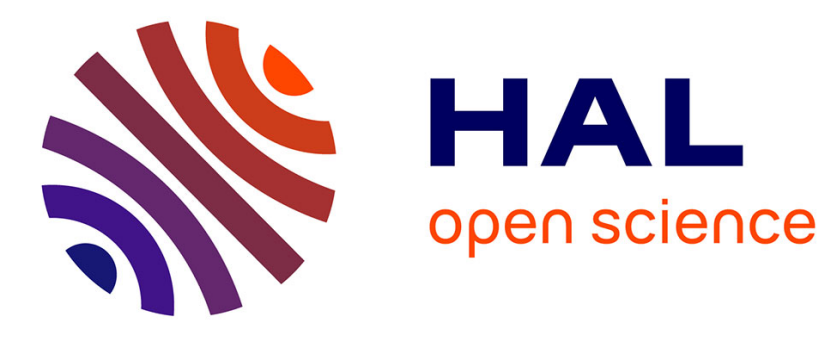

\title{
Analysing the Impact of Rationality on the Italian Electricity Market
}

Sara Bevilacqua, Célia da Costa Pereira, Eric Guerci, Frédéric Precioso, Claudio Sartori

\section{- To cite this version:}

Sara Bevilacqua, Célia da Costa Pereira, Eric Guerci, Frédéric Precioso, Claudio Sartori. Analysing the Impact of Rationality on the Italian Electricity Market. Modeling Decisions for Artificial Intelligence (MDAI 2019), Sep 2019, Milan, Italy. hal-02294953

\section{HAL Id: hal-02294953 \\ https://hal.science/hal-02294953}

Submitted on 23 Sep 2019

HAL is a multi-disciplinary open access archive for the deposit and dissemination of scientific research documents, whether they are published or not. The documents may come from teaching and research institutions in France or abroad, or from public or private research centers.
L'archive ouverte pluridisciplinaire HAL, est destinée au dépôt et à la diffusion de documents scientifiques de niveau recherche, publiés ou non, émanant des établissements d'enseignement et de recherche français ou étrangers, des laboratoires publics ou privés. 


\title{
Analysing the Impact of Rationality on the Italian Electricity Market
}

\author{
Sara Bevilacqua ${ }^{1}$, Célia da Costa Pereira ${ }^{2}{ }^{\star}$, Eric Guerci $^{3}$, Frédéric Precioso ${ }^{2}$, \\ and Claudio Sartori ${ }^{1}$ \\ 1 Università di Bologna \\ sara.bevilacqua, claudio.sartori@unibo.it \\ 2 Université Côte d'Azur, CNRS, I3S Lab. \\ celia.pereira,frederic.precioso@unice.fr \\ 3 Université Côte d'Azur, CNRS - GREDEG \\ eric.guerci@unice.fr
}

\begin{abstract}
We analyze the behavior of the Italian electricity market with an agent-based model. In particular, we are interested in testing the assumption that the market participants are fully rational in the economical sense. To this aim, we extend a previous model by considering a wider class of cases. After checking that the new model is a correct generalization of the existing model, we compare three optimization methods to implement the agents rationality and we verify that the model exhibits a very good fit to the real data. This leads us to conclude that our model can be used to predict the behavior of this market.
\end{abstract}

\section{Introduction and Related Work}

The need for understanding the evolution of the prices in the electrical power markets has increased with the new trends (the emergence of the liberalized market) of the electrical market in many countries [14,13]. The use of Artificial Intelligence techniques has already proven to be effective in modeling the electricity market. Faia et al. proposed in [3] a Genetic Algorithm (GA) based approach to solve the portfolio optimization problem for simulating the Iberian electricity market. The results show that their GA based method is able to reach better results than previous implementations of Particle Swarm Optimization (PS) and Simulated Annealing (SA) methods. Santos et al. proposed in [12] a new version of the Multi-Agent System for Competitive Electricity Markets (MASCEM, [11]) with the aim of optimizing it with repect to the results as well as to the execution time.

Other models have been proposed, like the one presented by Urielli et al. [17], in which the authors study the impact of the Time-Of-Use (TOU) tariffs in a competitive electricity market place. A very interesting and recent survey of potential design changes in the electricity market and their consequences, has been proposed by Ela et al. in [2].

* The second author acknowledges support of the project PEPS AIRINFO funded by the CNRS. 
In this paper, we propose a framework which helps analysing the behavior of the participants in the Italian electrical power market [15]. We would like to stress that our interest is in understanding how the market behaves as a consequence of the actions of its participants to make profit, and also in analysing the behavior of the market in order to maximize the social welfare from an economical rational point of view $[18,16]$, i.e., with respect to the electricity producers as well as with respect to the electricity consumers. To this aim, we first reproduce and then extend an existing economical-based model for the Italian electricity market [7].

The paper is organized as follows. Section 2 briefly presents the three optimization methods used in the paper. Section 3 presents the mechanism of exchanges in the Italian market proposed in the literature. Section 4 presents the exteded model as well as the obtained results. Section 5 concludes the paper.

\section{Some Background: A Brief Description of the used Methods}

In this section, we will briefly present the three methods used in our work to model the rationality of the market participants.

A Genetic Algorithm $(G A)[8,6]$ is a computational technique inspired by biology. The basic idea of a GA is to mimic the Darwinian principle of survival according to which species with a high capacity of adaptation have an higher probability to survive and then to reproduce. The algorithm considers a population of individuals represented by their genes. Three operators can be used to mimic the evolution of these individuals: mutation which randomly changes some bits of a gene, crossover which mimics the sexual reproduction of the living beings, and selection which consists of deciding which among the individuals in the population will survive in the next generation. This choice is made thanks to a fitness function which is an objective function allowing to compute the extent to which an individual of the population is adapted to solve the considered problem.

In Monte Carlo Optimization [1], an approximation to the optimum of an objective function is obtained by drawing random points from a probability distribution, evaluating them, and keeping the one for which the value of the objective function is the greatest (if a maximum is sought for) or the least (if a minimum is sought for). As the number of points increases, the approximation converges to the optimum.

Particle Swarm Optimization (PS) [10,9] is a meta-heuristic method inspired by the behavior or rules that guide the group of animals, for example bird flocks. According to these rules, the members of the swarm need to balance two opposite behaviors in order to reach the goal: individualistic behavior, in which each element searches for an optimal solution, and social behavior, which allows the swarm to be compact. Therefore, individuals take advantage from other searches moving toward a promising region. In this algorithm, the evolution of the population is re-created by the changing of the velocity of the particles. The idea is to tweak the values of a group of variables in order to make them become closer 
to the member of the group whose value is closest to the considered target. PS is similar to genetic algorithms (GAs). It is also a population-based method with the particularity that the elements of the population are iteratively modified until a termination criterion is satisfied.

\section{The Italian Electricity Market}

\subsection{The Market Configuration}

The reality of the Italian Electricity Market which takes place in the Italian Power Exchange (IPEX), considers a two-settlement market configuration with a generic forward market and the Day-Ahead Market (DAM). The DAM price value is commonly adopted as underlying for forward contracts; therefore, as in Guerci et al. [7], we will refer to DAM as the spot (i.e. immediate, instant) market session for simplicity. The forward market session is modeled by assuming a common, zone-independent, and unique forward market price $P^{f}$ for all market participants and by determining the exact historical quantity commitments for each generating unit.

\section{Definition 1 (Generating Company).}

A generating company (GenCo) is an agent $g$, (with $g=1,2, \ldots, G$, and $G$ is the number of GenCos) which owns $N_{g}$ generators ${ }^{4}$. The ith generator (where $\left.i=1,2, \ldots, N_{g}\right)$ has lower $\underline{Q}_{i, g}$ and upper $\bar{Q}_{i, g}$ production limits, which define the feasible production interval for its hourly real-power production level $\hat{Q}_{i, g, h}=$ $\hat{Q}_{i, g, h}^{f}+\hat{Q}_{i, g, h}^{s}([M W])$, with $\underline{Q}_{i, g} \leq \hat{Q}_{i, g, h} \leq \bar{Q}_{i, g}$ where $\hat{Q}_{i, g, h}^{f}$ and $\hat{Q}_{i, g, h}^{s}$ are respectively the quantity sold in the forward market and the quantity accepted in the $D A M$.

It is assumed that the company $g$ takes a long position in the forward market (it means that the company makes agreement with the market operator with large advance) for each owned generator $i$, corresponding to a fraction $f_{i, g, h}$ (where $h$ indicates the hour of the day) of its hourly production capacity, that is $\hat{Q}_{i, g, h}^{f}=f_{i, g, h} \cdot \bar{Q}_{i, g}$. The value of such fraction varies throughout the day, indeed forward contracts are commonly sold according to standard daily profiles. The value of $f_{i, g, h}$ has been estimated by looking at historical data and thus corresponds to a realistic daily profile for each generator.

Definition 2 (Revenues for the forward and spot markets). The revenue, $R_{g, h}^{f}([€ h])$, from forward contracts for company $g$ is:

$$
R_{g, h}^{f}=\sum_{i=1}^{N_{g}} \hat{Q}_{i, g, h}^{f} \cdot P^{f}
$$

The spot revenue, $R_{g, h}^{s}$, per hour for GenCo $g$ is obtained as follows:

\footnotetext{
${ }^{4}$ In the following we will use the terms generator and power plant interchangeably.
} 


$$
R_{g, h}^{s}=\sum_{z=1}^{Z} \hat{Q}_{z, g, h}^{s} \cdot P_{z, h}^{s}
$$

where $P_{z, h}^{s}$ is the price in the spot market in zone $z$ at hour $h$, and $Z$ is the total number of zones.

Let $C_{i, g, h}([€ / \mathrm{h}])^{5}$ be the total cost (of production) function of the $i^{\text {th }}$ generator of GenCo $g$. The total profit per hour, $\pi_{g, h}$, $[€ / \mathrm{h}]$ for GenCo $g$ is computed as follows:

$$
\pi_{g, h}=R_{g, h}^{s}+R_{g, h}^{f}-\sum_{i=1}^{N_{g}} C_{i, g, h}\left(\hat{Q}_{i, g, h}\right)
$$

The considered set of thermal power plants, independently owned by GenCos, consists of up to 224 generating units, using 5 different technologies. The number of generation companies and generating units offering in the DAM varies throughout the day. Based on historical data, it has been determined for each period (day and hour) the thermal power plants that offered in DAM. ${ }^{6}$ For each power plant in the dataset, information on the maximum and minimum capacity limits is available, as well as on the parameters needed to compute the cost.

\subsection{Market Exchanges}

A GenCo $g$ submits to the DAM a bid consisting of a pair of values corresponding to the limit price $P_{i}^{s}([€ / \mathrm{MW}])$ and the maximum quantity of power $Q_{i}^{s} \leq$ $\bar{Q}_{i, g}-\hat{Q}_{i, g}^{f}([\mathrm{MW}])$ that it is willing to be paid and to produce, respectively. After receiving all generators bids, the market operator clears the DAM by performing a social welfare maximization, subject to the following constraints:

- the zonal energy balance (Kirchhoffs laws),

- the maximum and minimum capacity of each power plant,

- the inter-zonal transmission limits.

It is worth noting that the Italian demand curve in the DAM is price-inelastic, i.e., it is unaffected when the price changes. Therefore, the social welfare maximization can be transformed into a minimization of the total reported production costs, i.e., of the bid prices (see Equation 4). This mechanism determines both the unit commitments for each generator and the Locational Marginal Price (LMP) for each connection bus. However, the Italian market introduces two slight modifications. Firstly, sellers are paid the zonal prices (LMP), therefore, this fact has to be explicitly considered in the model, whereas buyers pay a

\footnotetext{
5 The details about the function can be found in [7].

${ }^{6}$ Notice that bid data are publicly available on the power exchange website with a one-week delay, therefore, information about what plants were actually present and the like is supposed to be common knowledge.
} 
unique national price (PUN, Prezzo Unico Nazionale) common for the whole market and computed as a weighted average of the zonal prices with respect to the zonal loads. Secondly, transmission power-flow constraints differ according to the flow direction.

The factor which has to be minimized by solving the linear program is the following:

$$
\min \sum_{g=1}^{G} \sum_{i=1}^{N_{g}} P_{i, g, h}^{s} \hat{Q}_{i, g, h}^{s}
$$

It is subjected to the following constraints:

- Active power generation limits: $\underline{Q}_{i, g} \leq \hat{Q}_{i, g, h}=\hat{Q}_{i, g, h}^{s}+\hat{Q}_{i, g, h}^{f} \leq \bar{Q}_{i, g}[\mathrm{MW}]$

- Active power balance equations for each zone $z$ : $\sum_{g=1}^{G} \sum_{j \in z} \hat{Q}_{j, g, h}^{s}-Q_{z, \text { load }, h}=Q_{z, \text { inject }, h}[\mathrm{MW}]$ being $\sum_{g=1}^{G} \sum_{j \in z} \hat{Q}_{j, g, h}^{s}$ the sum of all the productions over all generators located in zone $z, Q_{z, l o a d, h}$, the load demand at zone $z$ in hour $h$ and $Q_{z, \text { inject, } h}$, the net oriented power injection in the network at zone $z$ in hour $h$.

- Real power flow limits of line, $l: Q_{l, s t} \leq \bar{Q}_{l, s t}[\mathrm{MW}]$ and $Q_{l, t s} \leq \bar{Q}_{l, t s}$ [MW] being $Q_{l, s t}$ the power flowing from zone $s$ to zone $t$ of line $l$ and $\bar{Q}_{l, s t}$ the maximum transmission capacity of line $l$ in the same direction. $Q_{l, s t}$ are calculated with the standard DC power flow model [4].

The solution consists of the set of the active powers $\hat{Q}_{i, g, h}^{s}$ generated by each plant $i$ and the set of zonal prices $P_{z}^{s}$ (LMPs) for each zone $z \in[1,2, \ldots, Z]$, where $Z$ is the number of zones.

\section{Relaxing the zonal constraint}

In this section we will present the new "relaxed" framework as well as the results we have obtained from our experiments.

\subsection{Model description}

Each GenCo $g$ must submit to the DAM a bid, i.e., a set of prices for each of its own power plants. Therefore, each GenCo has an action space for each power plant, which is a set of possible prices that the GenCo can choose. This set is represented by Vector $A S_{i, g}$ which is obtained with the following product:

$$
A S_{i, g}=M C_{i, g} \cdot M K s e t,
$$

where, $A S_{i, g}$ represents the action space of power plant $i$ of Genco $g, M C_{i, g}$ is the marginal cost of the same power plant, and $M K$ set $=[1.00,1.04, \ldots, 5.00]$ is the vector with the mark-up levels. In this way, GenCos are sure not to propose a price lower than the costs. 
The Multi-Agent System The multi-agent system is depicted in Figure 1. The $G$ GenCos are reported on the top of the figure. These GenCos repeatedly interact with each other at the end of each period $r \in\{1, \ldots, R\}$, that is they all submit bids to the DAM according to their current beliefs on opponents strategies. At the beginning of each period $r$, GenCos need to study the current

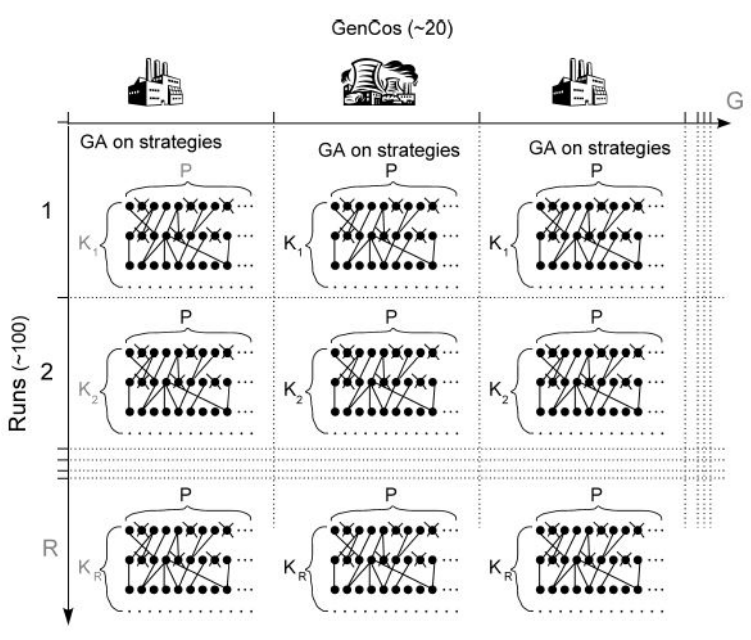

Fig. 1. A schematic representation of a simulation.

market situation in order to identify a better reply to the opponents, to be played at period $r+1$.

In order to choose the most competitive strategy, GenCos need to repeatedly solve the market for different private strategies. This correponds to an optimization problem.

\subsection{The Optimization Process}

In this context, the goal of the optimisation algorithm is to keep a large population of candidate strategies and to improve at the same time their fitness/performance in the market. Thus, a population of size $P$, (see Figure 1), of strategies is defined, which will evolve throughout the $K_{r}$ generations. Unlike Guerci et al. [7], we consider that each power plant can have its own strategy independently of the zone.

A strategy In this new framework, a strategy for a GenCo $g$, depicted as a black dot in Figure 1, is a vector of prices in the action space, one for each of the $N_{g}$ power plants of GenCo $g$. 
The two used fitness functions In [7], for a given GenCo $g$, a fitness function, $f_{g}: \mathbb{R}^{A_{g}} \rightarrow \mathbb{R}$ returns the profit of a strategy which is a vector of prices in the action space, one for each collection of power plants, $\left(A_{g}\right)$, situated in the same zone and using the same technology. As it has been defined in [7], such a fitness reinforces the weight of the power plants with low prices. We will name it type 1 fitness.

In our "relaxed" framework, instead, for a given GenCo $g$, a fitness function, $f_{g}^{\prime}: \mathbb{R}^{N_{g}} \rightarrow \mathbb{R}$ returns the profit of a strategy which is a vector of prices in the action space, one for each single power plant. Here, we propose a more "realistic" fitness which considers the amount of profit (given by Equation 3) a given individual (strategy) allows the power plans to get. We will name it type 2 fitness.

Selecting a Strategy At the end of each period $r$, each GenCo bids to the market by selecting one strategy belonging to its current population of candidates.

In [7] the selection is done according to a probabilistic choice model in order to favor the most represented strategy in the population (i.e., based on the frequency probability). Here, in addition to the frequency based strategy of selection used in [7], we use a second strategy based on the value of the fitness of the individuals. We name it fitness-based strategy.

\subsection{Evaluation of the Proposed Approach}

We have considered and tested two different market models:

- ApproxGenco, which replicates the market model proposed in [7] — the GenCos adopt a common strategy for all the power plants with a common technology, situated in the same zone.

- RealGenco, which relaxes the above constraint and makes it possible to model the fact that each power plant may adopt a different strategy — this is the way it happens in the reality.

The goal of the experiments We have evaluated the effectiveness of the proposed framework. To this aim we have:

- shown that our model really extends the model proposed in [7],

- verified if the fact of relaxing the assumptions according to which all the power plants in the same zone should be associated to the same strategy compromises the results,

- compared the results obtained with a simplest statistical method relying on repeated random sampling as the Monte Carlo method with the results obtained with the two studied models,

- verified if another population based-method like the Particle Swarm Optimisation (PS) method, lying on the hypothesis of cooperation between the 
individuals in the population, instead of Genetic Algorithms (GA) in which the individuals of the population can be perceived as being in competition, may improve the results.

The Different Scenarios In order to test the ability and the robustness of each optimization method to reproduce the daily PUN time series, we have considered the three methods (Genetic Algorithm, Particle Swarm, Montecarlo), the two fitness functions, (type 1 and type 2 fitness) and the two types of strategy choice (probability based and fitness based), obtaining thus the different scenarios listed in Table 1.

Table 1. Experimental scenarios

\begin{tabular}{|c|c|c|}
\hline Acronym & $\begin{array}{c}\text { Fitness function of } \\
\text { the genetic algorithm }\end{array}$ & $\begin{array}{c}\text { Best strategy choice } \\
\text { based on }\end{array}$ \\
\hline GaFreq1 & Type 1 & Frequency \\
\hline GaFreq2 & Type 2 & Frequency \\
\hline GaFitness1 & Type 1 & Fitness \\
\hline GaFitness2 & Type 2 & Fitness \\
\hline PsFreq1 & Type 1 & Frequency \\
\hline PsFreq2 & Type 2 & Frequency \\
\hline PsFitness1 & Type 1 & Fitness \\
\hline PsFitness2 & Type 2 & Fitness \\
\hline Montecarlo & Type 2 & Fitness \\
\hline
\end{tabular}

Data The demand of energy for each zone is provided in a load matrix with the following information: a first column which contains the zones, the second which contains the maximum limit prices and the third column which contains the demand quantities of electricity.

All the characteristics of the power plants are collected in a structure with the following features:

- the names of the GenCos (for example ATEL, EDISON, ...),

- the names of the used technologies (for example coal, combined cycle gas turbine,...),

- the prices of the fuels,

- information related to the Italian power plants: the columns indicates respectively the zone, maximum production quantity, minimum production quantity, coefficient $a$, coefficient $b$, coefficient $c$ (see 3), Genco's id, technology index, and fuel index and power plant's id. 
- the production quantity data from other power plants (i.e. not produced by the GenCo).

The PUN historical values used in the experiments are public data which can be found in [5].

Implementation and Results The framework described has been implemented in MATLAB R2017a with Optimization and Global Optimization toolboxes. Experiments were performed on a computer running Windows 7 and based on an IntelCoreTMi7-3610QM @2.30GHz microprocessor with 8 GB RAM.

In all the simulations, the number of GenCos participating in the market varies between 15 and 19, while the number of power plants for each GenCo varies between 1 and 90. The three optimisation methods used the matlab default parameters and they are allocated the same number of objective function evaluations.

The results of our experiments lead us to conclude that the ApproxGenco model can be considered as a reliable replication of the model proposed in [7]. Indeed, under the same conditions (the ones supported by the old model), it reproduces exactly the same market mechanism - we obtain the same result, i.e., the same PUN with the two models.

In Figure 2, we can see the historical values (red line), as well as the values obtained with [7] (we named it old) which are represented with dashed (purple) lines and the values obtained with our relaxed model under the same assumptions that the ones made in [7] (we named it GAfreq1, they are represented by the green line with squares).

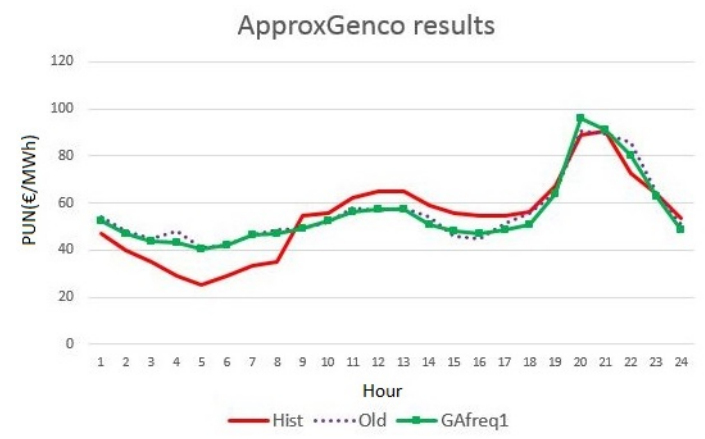

Fig. 2. Reproducibility - Real and simulated PUNs provided by ApproxGenco.

We can now proceed with its comparison with the alternative scenarios.

Two interesting points emerge. We can observe, thanks to the results illustrated in Figure 3, that despite having "relaxed" the constraint reducing the action space, and under the same assumptions, (i) the results are similar for both ApproxGenco and RealGenco models, i.e., the simulated PUN is similar, (ii) 
the time needed for running the ApproxGenco and RealGenco models is similar. More precisely, the running time of each iteration for ApproxGenco with a population of 50 elements is 11.62 seconds for GA, 15.76 seconds for PS, and 11.34 seconds for Monte Carlo. Concerning the RealGenco the values are: 14.56 seconds for GA, 18.68 seconds for PS and 10,5 seconds for Monte Carlo.

We can then conclude that we can take into account the differences between the power plants as for example their different efficiency levels without worsening the quality of the results.

Evaluation of the RMSD. These considerations at macro-level are supported by the evaluation of the root-mean-square deviation (RMSD) which is a frequently used measure of the difference between values predicted by a model and the values actually observed. The RMSD represents the sample standard deviation of the differences between predicted values and observed values. The formula we have used is the following:

$$
R M S D=\sqrt{\frac{\sum_{h=1}^{24}\left(\hat{y}_{h}-y_{h}\right)^{2}}{24}}
$$

where $h$ represents the hour of the day (therefore it varies between 1 and 24 ), $\hat{y}_{h}$ and $y_{h}$ are respectively the predicted value and the observed value of the PUN at hour $h$.

Figure 3 shows the RMSD of all the scenarios we have considered for ApproxGenco's and RealGenco's.

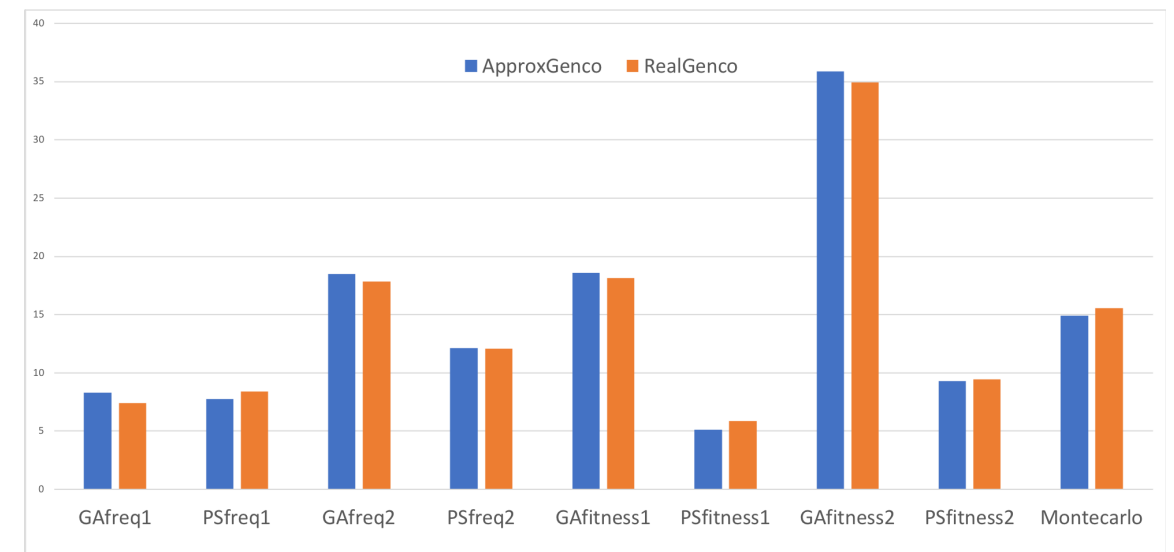

Fig. 3. RMSD for ApproxGenco and RealGenco methods

Evaluation of the absolute error. These considerations at macro-level are also supported by the results concerning the absolute error which, in our work, rep- 
resents the distance between the curve with the historical PUNs values and the one obtained from the different scenarios. The formula used is the following:

$$
A E_{h}=\left|p r_{h}-h i s t_{h}\right|
$$

where $p r_{h}$ is the prediction at hour $h$ and $h i s t_{h}$ is the historical value of the PUN at the same hour.

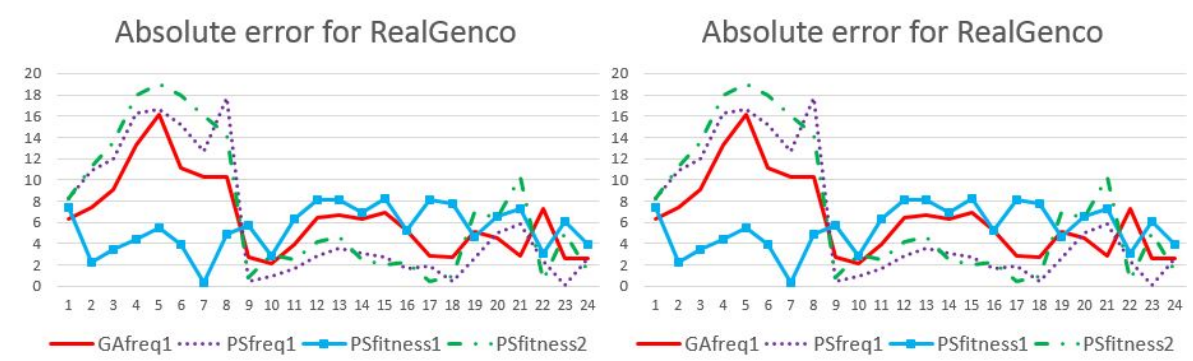

Fig. 4. Absolute errors for the best ApproxGenco model and for the RealGenco model.

Figure 4 illustrates the absolute errors for the three best optimization methods for both ApproxGenco and RealGenco models. We can notice that in the ApproxGenco model the results obtained by the three PS algorithms are better than the ones obtained by the best GA algorithm in the peak hours. Instead, during the off-peak hours the GA algorithm outperforms two out of three PS algorithms. Things are slightly different with the RealGenco model, where PSfitness1 (cyan line with squares) outperforms all the other algorithms in the off-peak hours while it produces the worse results in the peak hours. However, the PSfreq1 algorithm (dashed purple line) outperforms all the others in the peak hours.

\section{Conclusion}

We have extended an existing agent-based model of the Italian electricity market and we have investigated the rationality of the market participants by comparing three optimization methods.

We can conclude that in the reality the planning for managing GenCos follows a rational strategy which can be modeled thanks to an optimization method without reducing the action space. We can also conclude that the particle swarm optimization method is the method which allows to better simulate the behavior of the agents in the Italian electricity market — its results better fit with the historical PUN values. Therefore, our model can be used to predict the behavior of the Italian electricity market, for example by performing counterfactual analyses. 


\section{References}

1. B. Betrò, M. Cugiani, and F. Schoen. Monte Carlo Methods in Numerical Integration and Optimization. Applied Mathematics Monographs, CNR. Giardini, Pisa, 1990.

2. E. Ela, C. Wang, S. Moorty, K. Ragsdale, J. OSullivan, M. Rothleder, and B. Hobbs. Electricity markets and renewables. IEEE Power and Energy Magazine, 15(27):1540-7977, 2015.

3. R. Faia, T. Pinto, and Z. A. Vale. GA optimization technique for portfolio optimization of electricity market participation. In 2016 IEEE Symposium Series on Computational Intelligence, SSCI 2016, Athens, Greece, December 6-9, 2016, pages $1-7,2016$.

4. G. Giulioni, C. Hernández, M. Posada, and A. López-Paredes. Artificial Economics: The Generative Method in Economics. Lecture Notes in Economics and Mathematical Systems 631. Springer-Verlag Berlin Heidelberg, 1 edition, 2009.

5. GME. http://www.mercatoelettrico.org/it/download/datistorici.aspx.

6. D. E. Goldberg. Genetic Algorithms in Search, Optimization 83 Machine Learning. Addison-Wesley, Reading, MA, 1989.

7. E. Guerci, M.A. Rastegar, and S. Cincotti. Agent-based modeling and simulation of competitive wholesale electricity markets. In Steffen Rebennack, Panos M. Pardalos, Mario V. F. Pereira, and Niko A. Iliadis, editors, Handbook of Power Systems II, pages 241-286. 2010.

8. J. H. Holland. Adaptation in Natural and Artificial Systems. The University of Michigan Press, Ann Arbor, 1975.

9. J. Kennedy. Particle swarm optimization. In Encyclopedia of Machine Learning and Data Mining, pages 967-972. Springer, 2017.

10. J. Kennedy and R. Eberhart. Particle swarm optimization. In Proceedings of IEEE International Conference on Neural Networks, Part IV, pages 1942-1948, 1995.

11. T. Pinto, H. Morais, P. Oliveira, Z. Vale, I. Praça, and C. Ramos. A new approach for multi-agent coalition formation and management in the scope of electricity markets. Energy, 36(8):5004 - 5015, 2011.

12. G. Santos, T. Pinto, I. Praça, and Z. Vale. Mascem: Optimizing the performance of a multi-agent system. Energy, 111(Supplement C):513 - 524, 2016.

13. F. Silva, B. Teixeira, T. Pinto, G. Santos, I. Praça, and Z.A. Vale. Demonstration of realistic multi-agent scenario generator for electricity markets simulation. In Advances in Practical Applications of Agents, Multi-Agent Systems, and Sustainability: The PAAMS Collection - 13th International Conference, PAAMS 2015, Salamanca, Spain, June 3-4, 2015, Proceedings, pages 316-319, 2015.

14. F.P. Sioshansi. Academic Press, Boston, 2013.

15. C. Tribbia. Solving the italian electricity power exchange, 2015.

16. D. Urieli. Autonomous trading in modern electricity markets. AI Matters, 2(4):1819, 2016.

17. D. Urieli and P. Stone. Autonomous electricity trading using time-of-use tariffs in a competitive market. In Proceedings of the Thirtieth AAAI Conference on Artificial Intelligence, February 12-17, 2016, Phoenix, Arizona, USA., pages 345-352, 2016.

18. P. Vytelingum, S. D. Ramchurn, T. Voice, A. Rogers, and N.R. Jennings. Trading agents for the smart electricity grid. In $A A M A S$, pages 897-904. IFAAMAS, 2010. 